\title{
Analysis of advection-diffusion-reaction model for fish population movement with impulsive tagging: stability and traveling wave solution
}

\author{
Chontita Rattanakul ${ }^{1,3}$, Yongwimon Lenbury ${ }^{1,3^{*}}$ (D) and Jeerawan Suksamran ${ }^{2,3}$
}

\author{
"Correspondence: \\ yongwimon.len@mahidol.ac.th \\ 1 Department of Mathematics, \\ Faculty of Science, Mahidol \\ University, Bangkok, Thailand \\ ${ }^{3}$ Centre of Excellence in \\ Mathematics, CHE, Bangkok, \\ Thailand \\ Full list of author information is \\ available at the end of the article
}

\begin{abstract}
So far, mobility in fish population has not been given sufficient attention, although movement and spatial heterogeneity can be an important dynamic feature that plays a critical role in their exploitation. Having a qualitative framework to describe and estimate movement and growth based on tagging data is necessary for efficient control and management of the fishing industry. Here, we construct an advection-diffusion-reaction model for the fish population structured to track the population densities of both the tagged fish and the tag-free fish, in which the impulsive tagging practice is incorporated, while continuous tagging is assumed to be done on off springs of trackable tagged fish, or on those in the same swarm as the trackable tagged fish. Using the traveling wave coordinate, we derive analytical expression for the solutions to the model system. We derive the explicit expression for the level of tagged fish which increases in a periodic impulsive fashion. Stability and phase plane analyses are also carried out to determine different behavior permitted by the model system.
\end{abstract}

Keywords: Advection-diffusion-reaction model; Fish mobility; Traveling wave solution; Stability analysis; Impulsive tagging

\section{Introduction}

Migration that involves movements among different habitats of is a common characteristics of marine and freshwater fish populations. They are large in spatial scale compared to their movements within the home range of the aquatic species [1]. As stated in [1], migration is an important and integral element of the life history of many fish species in aquatic systems. The manner in which fish migrates is often linked to the necessity of seeking resources such as food, shelter, or mates [1]. According to Sibert et al. [2], a crucial aspect of fisheries is that the constituents are highly heterogeneous nature in space and time. This feature significantly effects their management, so that fish mobility and distribution have to be precisely described using appropriate models that take into account both spatial and temporal variations. Marine populations can be overexploited if management decisions do not account for spatial structuring [3-5]. However, although spatial structuring is a

(c) The Author(s) 2019. This article is distributed under the terms of the Creative Commons Attribution 4.0 International License (http://creativecommons.org/licenses/by/4.0/), which permits unrestricted use, distribution, and reproduction in any medium, provided you give appropriate credit to the original author(s) and the source, provide a link to the Creative Commons license, and indicate if changes were made. 
common aspect of fish life history, it is often overlooked or omitted in modeling of fish population dynamics due to insufficient data or lack of understanding $[4,6,7]$.

It has recently become more common that our knowledge of long distance movements of animals comes from tagging studies. With fish spatial distribution, it is beyond current technology to track individuals very far, so that tagging data tell us where tagged fish were released and where they were recovered. Knowledge of the movement patterns of populations or individuals is sought by the fisheries managers and biologists in order to assess the interaction between aquatic species in different spatial locations and to define the functioning of stocks. According to Hilborn [8], tagging studies, though, are often the only way to assess total stock size.

The two strategies used routinely to assess exploited fish stocks are statistical catch-atage (SCAA) and tag-recovery models [9]. In [10], Pine et al. gave a brief review of population models that can be used to estimate population size, with the intention that fisheries biologists may be assisted in designing tagging studies by considering the underlying assumptions, available basic models and specialized software.

In 2001, the movement and tag attrition parameters from skipjack tuna tagging data off the Maldives was modeled by an advection-diffusion-reaction model, utilizing two sets of field data collected during two periods of the early 1990s [11]. When their analytical results were compared with the previous analyses in regards to skipjack fisheries management in the Maldives and in the Indian Ocean, it was discovered that the movements were very variable in space and time, and it was difficult to observe consistent patterns between the two data sets. In 2002, Bertignac et al. [12] developed a spatial, multigear, multispecies population dynamics model which simulated the tropical tunas in the Pacific Ocean. It includes a fish movement model based on a diffusion-advection equation. The simulation model was shown to be capable of predicting a distribution of skipjack catch rates, of the different fleets involved in the fisheries that is consistent with observation. In 2005, Faugeras and Maury [13] proposed an advection-diffusion size-structured fish population model to simulate the dynamics of skipjack tuna population in the Indian Ocean. Their model is fully spatialized and oceanographic and biological data were utilized to parameterize movements which naturally react to environmental variations. More recently, Boonrangsiman et al. [14] proposed a stage-structure model of fisheries which incorporated a time delay. The single prey population and a predator population were differentiated with respect to their reproduction ability into an immature and a mature stage. Steady state analyses were carried out. It was shown that the steady state may lose its stability under certain conditions and a Hopf bifurcation may take place at a critical time delay. The model was shown to permit a transition to chaotic behavior.

With respect to traveling wave solutions in models of fisheries, we discovered that, in [15], a simple model was introduced. The direction in which organisms prefer to move is guided by their sensing. They showed that the model has a one parameter family of compact traveling waves or "swarms". Also, the model possesses traveling front solutions that describe the migration of population from a region in which the population density is higher to that where the density is lower. Notably, their model simulates realistic features of organism aggregations whose speed increases with the density which has been observed in fish schools.

To our knowledge, none of the previous advection-diffusion-reaction models have been structured into populations of tagged and untagged fisheries. Incorporation of these dis- 
tinctive characteristics could lead to greater accuracy and precision of stock size estimates from tagging studies and thus improve understanding and management of fisheries. Here, we therefore propose an advection-diffusion-reaction model to describe fish movement, tracking population densities of tagged fish and untagged fish. This is extended to model periodic tagging, leading us to an impulsive advection-diffusion-reaction model system. We first derive analytical solutions for the model system during the time between consecutive tagging. By introducing traveling wave coordinate, we derive an equivalent system of ordinary differential equations whose analytical solution can be found. This analytical solution can then be modified to take into account periodic tagging. Stability analysis is also carried out after which plots of traveling wave solutions are shown and discussed.

\section{Model system}

We let $T(x, t)$ and $N(x, t)$ be, respectively, numbers per unit area (densities) of tagged and untagged fish populations at the time $t$ and spatial position $x$. Incorporating fish movement with a diffusion term, we write the following advection-diffusion-reaction equations for the rates of change of $T(x, t)$ and $N(x, t)$ under impulsive tagging:

$$
\begin{aligned}
& \left.\begin{array}{rl}
\frac{\partial T}{\partial t}= & \frac{\partial}{\partial x}\left(\rho \frac{\partial T}{\partial x}\right)-\frac{\partial}{\partial x}(u T)-d_{0} T+b_{T} T, \\
\frac{\partial N}{\partial t}= & \frac{\partial}{\partial x}\left(\rho \frac{\partial N}{\partial x}\right)-\frac{\partial}{\partial x}(u N)-d_{0} N-b_{T} T \\
& +b_{S}(T+N)\left(\varepsilon_{S}-(T+N)\right)
\end{array}\right\} t \neq k \Delta t, \\
& \left.\begin{array}{l}
\Delta T\left(0, t^{+}\right)=p N(0, t), \\
\Delta N\left(0, t^{+}\right)=-p N(0, t)
\end{array}\right\} \quad t=k \Delta t, k=1,2,3, \ldots,
\end{aligned}
$$

where $\rho$ is the diffusion coefficient, assumed to be a positive constant. The first terms on the right of (1) and (2) thus model the dispersive movement of fish as a diffusion process. The second terms on the right of (1) and (2) are added to account for the "directed" movement as an advection process. $d_{0}$ is the motility rate constant for both populations, and $b_{s}$ corresponds to the rate of birth of untagged fish from $S(x, t)=T(x, t)+N(x, t)$, the sum of tagged and untagged fish populations, modeled by a logistic growth function, $\varepsilon_{S}$ being the carrying capacity of the environment. Apart from the periodic tagging described by Eq. (3), the untagged fish individuals are assumed to be continuously tagged at the rate $b_{T} T$ which takes into account of the possibility that the tagged individuals can attract untagged fish to move together in a directional fashion within the same swarm and hence the untagged fish become caught and tagged at a higher rate. In addition, off springs of tagged fish may be more readily tagged at the rate proportional to the amount of tagged fish at each moment in time. Realistically, this tagging effect is a function which may also depend on spatial distance and time. However, we are using the linear expression $b_{T} T$ as the first order approximation of this function, assuming that the higher order terms in its expansion are much smaller, and let the dimensions of distance and time be taken into account by $T$ which is expected to become smaller as we travel further along downstream.

To support the above assumption, we refer to [16], where Birnir and Maury observe that the intrinsic dynamics of a school of fish and its migration is a perplexing and fascinating phenomenon which presents us with many complications in our attempts at optimization 
and management. This is essentially due to the fact that individual fish has the tendency to adjust their speed and movement direction to those of the school to which they belong. The fish population organizes and maintains schools from a basic mechanism in predator avoidance and survival tactics. The extraordinary speed at which individual fish reacts in unison to predatory attacks appears to result from the quick transfer of information locally among members of a school. These groups turn together collectively and produce "escape waves" [16].

In (3), at the location $x=0$, a fraction $p$ of untagged fish is recruited and tagged, thereby increasing the density of tagged fish by $p N(t, 0)$ every time period of $\Delta t$.

We first consider the time between tagging, $t \neq k \Delta t, k=1,2,3, \ldots$. The model of interest in this case then consists of the following equations:

$$
\begin{aligned}
& \frac{\partial T}{\partial t}=\frac{\partial}{\partial x}\left(\rho \frac{\partial T}{\partial x}\right)-\frac{\partial}{\partial x}(u T)-d_{0} T+b_{T} T \\
& \frac{\partial N}{\partial t}=\frac{\partial}{\partial x}\left(\rho \frac{\partial N}{\partial x}\right)-\frac{\partial}{\partial x}(u N)-d_{0} N-b_{T} T+b_{S}(T+N)\left(\varepsilon_{S}-(T+N)\right) .
\end{aligned}
$$

Adding (1) and (2), one obtains

$$
\frac{\partial S}{\partial t}=\frac{\partial}{\partial x}\left(\rho \frac{\partial S}{\partial x}\right)-\frac{\partial}{\partial x}(u S)-d_{0} S+b_{S} S\left(\varepsilon_{S}-S\right) .
$$

\subsection{Traveling wave coordinate}

We now introduce the traveling wave coordinate

$$
z=x-c\left(t-t_{0}\right)
$$

assuming that the wave of fish movement is traveling at a constant speed $c, t_{0}$ being the time of interest. Letting $\tau(z)=T(x, t), n(z)=N(x, t), s(z)=S(x, t)$, and $(\cdot)^{\prime}$ stands for the derivative with respect to $z$, we have

$$
\frac{\partial S}{\partial x}=s^{\prime}, \quad \frac{\partial^{2} S}{\partial x^{2}}=s^{\prime \prime}, \quad \frac{\partial S}{\partial t}=-c s^{\prime},
$$

and

$$
\frac{\partial T}{\partial x}=\tau^{\prime}, \quad \frac{\partial^{2} T}{\partial x^{2}}=\tau^{\prime \prime}, \quad \frac{\partial T}{\partial t}=-c \tau^{\prime} .
$$

Thus, Eq. (4) becomes

$$
\rho s^{\prime \prime}+(c-u) s^{\prime}+\left(b_{S} \varepsilon_{S}-d_{0}\right) s-b_{S} s^{2}=0
$$

while (1) becomes

$$
\rho \tau^{\prime \prime}+(c-u) \tau^{\prime}+\left(d_{0}+b_{T}\right) \tau=0 .
$$




\section{Analytical solutions}

During $k \Delta t<t<(k+1) \Delta t, k=1,2,3, \ldots$, we seek a traveling wave solution in the form

$$
s^{\prime}=B s^{m}-A s,
$$

where $m, A$, and $B$ are positive constants, which gives

$$
s^{\prime \prime}=\left(m B s^{m-1}-A\right)\left(B s^{m}-A s\right)=m B^{2} s^{2 m-1}+A^{2} s-(A B+m A B) s^{m} .
$$

Substituting (7)-(8) into (5), one obtains

$$
\rho\left(A^{2} s-(A B+m A B) s^{m}+m B^{2} s^{2 m-1}\right)+(c-u)\left(B s^{m}-A s\right)+\left(b_{S} \varepsilon_{S}-d_{0}\right) s-b_{S} s^{2}=0 .
$$

For a traveling wave solution to exist, we see that we must set $m=3 / 2$. Then, equating coefficients of like terms, we obtain

$$
\rho A^{2}-(c-u) A+\left(-d_{0}+b_{S} \varepsilon_{S}\right)=0
$$

from the coefficients of $s$,

$$
-5 \rho A+2(c-u)=0
$$

from the coefficients of $s^{3 / 2}$, and

$$
3 \rho B^{2}-2 b_{s}=0
$$

from the coefficients of $s^{2}$. Solving (10), one obtains

$$
A=\frac{2(c-u)}{5 \rho}
$$

since $\rho$ is a positive constant. Upon substituting into (9) into (12), one obtains

$$
-\frac{6(c-u)^{2}}{25 \rho}+\left(-d_{0}+b_{S} \varepsilon_{S}\right)=0
$$

noting here that we need

$$
b_{S} \varepsilon_{S}>d_{0} .
$$

Now, to solve (6), we seek a solution of the form

$$
\tau=\left(C z+\tau_{0}\right) e^{-D z}, \quad \tau_{0}=\tau(z=0)=T\left(0, t_{0}\right),
$$

where $C$ and $D$ are positive constants, for which

$$
\begin{aligned}
& \tau^{\prime}=-D \tau+C e^{-D z} \\
& \tau^{\prime \prime}=-D \tau^{\prime}-C D e^{-D z}=-D\left(-D \tau+C e^{-D z}\right)-C D e^{-D z}=D^{2} \tau-2 C D e^{-D z} .
\end{aligned}
$$


Substituting (14)-(16) into (6) yields $\rho\left(D^{2}\left(C z+\tau_{0}\right) e^{-D z}-2 C D e^{-D z}\right)+(c-u)(-D(C z+$ $\left.\left.\tau_{0}\right) e^{-D z}+C e^{-D z}\right)+\left(-d_{0}+b_{T}\right)\left(C z+\tau_{0}\right) e^{-D z}=0$. Equating coefficients of the terms $e^{-D z}$ in the above equation yields

$$
\rho D^{2} \tau_{0}-2 d C D+(c-u)\left(C-D \tau_{0}\right)+\left(-d_{0}+b_{T}\right) \tau_{0}=0
$$

Equating coefficients of the terms $z e^{-D z}$ yields

$$
\rho C D^{2}-(c-u) C D+\left(-d_{0}+b_{T}\right) C=0
$$

or

$$
\rho D^{2} \tau_{0}-(c-u) D \tau_{0}+\left(-d_{0}+b_{T}\right) \tau_{0}=0 .
$$

Subtracting (18) from (17) gives

$$
-2 \rho C D+(c-u) C=0
$$

or

$$
D=\frac{c-u}{2 \rho}
$$

while (18) then gives

$$
-d_{0}+b_{T}=\rho D^{2} \text {. }
$$

We note that, since $D>0$, we must set

$$
c>u
$$

and

$$
b_{T}>d_{0} .
$$

Using (19) and (20) in (13), one obtains a simplified relation as follows:

$$
b_{S} \varepsilon_{S}=b_{T}-\frac{\rho D^{2}}{25} \text {. }
$$

\subsection{Traveling wave solution}

To now derive the traveling wave solution for $s$, we re-write Eq. (7) as

$$
\int \frac{s^{\prime}}{B s^{3 / 2}-A s} d z=\int d z
$$

Letting $\mu=s^{1 / 2}$, the above integral may be written as

$$
\int \frac{2 \mu d \mu}{\left(B \mu^{3}-A \mu^{2}\right)}=\int \frac{2 d \mu}{\mu(B \mu-A)}=\int d z .
$$


Upon using partial fraction decomposition, we can carry out the integral to obtain

$$
\ln \left|\frac{B s^{1 / 2}-A}{s^{1 / 2}}\right|=\frac{A}{2}\left(z-z_{0}\right)
$$

or

$$
\frac{\left|A-B s^{1 / 2}\right|}{s^{1 / 2}}=\kappa e^{A z / 2},
$$

where

$$
\kappa=\frac{\left|A-B s_{0}^{1 / 2}\right|}{s_{0}^{1 / 2}},
$$

with $s_{0}=s(z=0)=S\left(x=0, t=t_{0}\right)$. To find a wave front solution of (6) as a decreasing function of $z$, we assume that $A>B s_{0}^{1 / 2}$, and then the solution is

$$
s=\frac{A^{2} e^{-A z}}{\left(\kappa+B e^{-A z / 2}\right)^{2}} .
$$

Thus, from (14) and (25), we obtain

$$
\begin{aligned}
& S(x, t)=\frac{A^{2} e^{-A\left(x-c\left(t-t_{0}\right)\right)}}{\left(\kappa+B e^{-A\left(x-c\left(t-t_{0}\right)\right) / 2}\right)^{2}}, \\
& T(x, t)=\left(C\left(x-c\left(t-t_{0}\right)\right)+\tau_{0}\right) e^{-D\left(x-c\left(t-t_{0}\right)\right)}, \\
& N(x, t)=S(x, t)-T(x, t),
\end{aligned}
$$

where the parameters are related according to (11), (12), (19), (20), and (23), subject to the conditions (21) and (22) as well as $b_{S} \varepsilon_{S}>d_{0}$.

\section{Stability analyses during no tagging}

We now first consider each separate state variable, $\tau(z)$ then $s(z)$.

\subsection{Tagged population}

During $k \Delta t<t<(k+1) \Delta t, k=1,2,3, \ldots$, we introduce in (6) the new variables: $X=\tau(z)$, and $Y=\tau^{\prime}(z)$, which lead us to the following system of first order nonlinear equations in $X$ and $Y$ :

$$
\begin{aligned}
& X^{\prime}=Y, \\
& Y^{\prime}=\frac{d_{0}-b_{T}}{\rho} X-\frac{c-u}{\rho} Y .
\end{aligned}
$$

The only steady state of $(29)-(30)$ is $\left(X_{0}, Y_{0}\right)=(0,0)$. We have the following result.

Theorem 1 For the system (29)-(30), we have the following.

1. If (21) and (22) hold, the steady state $\left(X_{0}, Y_{0}\right)=(0,0)$ of $(29)-(30)$ is locally asymptotically stable. 
2. If (21) or (22) is violated, such that

$$
c<u
$$

or

$$
d_{0}-b_{T}>0
$$

then $\left(X_{0}, Y_{0}\right)=(0,0)$ is unstable.

Proof The Jacobian matrix of (29)-(30) at $\left(X_{0}, Y_{0}\right)$ is

$$
J\left(X_{0}, Y_{0}\right)=\left(\begin{array}{cc}
0 & 1 \\
\frac{d_{0}-b_{T}}{\rho} & -\frac{c-u}{\rho}
\end{array}\right) .
$$

If (20) and (21) hold, we have

$$
\operatorname{Trace} J\left(X_{0}, Y_{0}\right)=-\frac{c-u}{\rho}<0
$$

and

$$
\operatorname{Det} J\left(X_{0}, Y_{0}\right)=-\frac{d_{0}-b_{T}}{\rho}>0
$$

Hence, $\left(X_{0}, Y_{0}\right)$ is locally asymptotically stable.

On the other hand if we have (31) while (22) holds, Trace $J\left(X_{0}, Y_{0}\right)>0$, and $\left(X_{0}, Y_{0}\right)$ is a saddle point or an unstable focus. If conditions (32) and (21) hold, then $\left(X_{0}, Y_{0}\right)$ is a saddle point, which is unstable.

\subsection{Total fish density}

During $k \Delta t<t<(k+1) \Delta t$, we introduce in (5) the new variables: $U=s(z)$, and $V=s^{\prime}(z)$, which leads us to the following system of first order nonlinear equations in $U$ and $V$ :

$$
\begin{aligned}
& U^{\prime}=V, \\
& V^{\prime}=\frac{d_{0}-b_{S} \varepsilon_{S}}{\rho} U+\frac{b_{S}}{\rho} U^{2}-\frac{c-u}{\rho} V .
\end{aligned}
$$

If

$$
b_{S} \varepsilon_{S}-d_{0}>0
$$

there are two physically meaningful steady states, $\left(U_{0}, V_{0}\right)=(0,0)$ and $\left(U_{1}, V_{1}\right)=\left(\frac{b_{S} \varepsilon_{S}-d_{0}}{b_{S}}\right.$, $0)$. We can state and prove the following stability result:

Theorem 2 For the system (33)-(34), if (21), and (35) hold, then:

1. $\left(U_{0}, V_{0}\right)$ is locally asymptotically stable.

2. $\left(U_{1}, V_{1}\right)$ is an unstable saddle point. 
Proof The Jacobian matrix of $(33)-(34)$ at $(0,0)$ is

$$
J(0,0)=\left(\begin{array}{cc}
0 & 1 \\
\frac{d_{0}-b_{S} \varepsilon_{S}}{\rho} & -\frac{c-u}{\rho}
\end{array}\right) .
$$

So, we have

$$
\operatorname{Trace} J\left(U_{0}, V_{0}\right)=-\frac{c-u}{\rho}<0
$$

and

$$
\operatorname{Det} J\left(U_{0}, V_{0}\right)=-\frac{d_{0}-b_{S} \varepsilon_{S}}{\rho}>0
$$

which means the eigenvalues have negative real parts and so $\left(U_{0}, V_{0}\right)$ is asymptotically stable. At $\left(U_{1}, V_{1}\right)$, the Jacobian is

$$
J\left(U_{1}, V_{1}\right)=\left(\begin{array}{cc}
0 & 1 \\
\frac{-d_{0}+b_{S} \varepsilon_{S}}{\rho} & -\frac{c-u}{\rho}
\end{array}\right) .
$$

Then

$$
\operatorname{Det} J\left(U_{0}, V_{0}\right)=-\frac{-d_{0}+b_{S} \varepsilon_{S}}{\rho}<0 \text {. }
$$

Hence, we conclude that $\left(U_{1}, V_{1}\right)$ is a saddle point, which completes the proof.

Combining the analyses of both 2 dimensional systems without impulsive tagging, we may conclude that, if (21), (22) and (35) hold, its washout solution $(0,0,0,0)$ is asymptotically stable as $z \rightarrow \infty$, while the solution $\left(0,0, \frac{b_{S} \varepsilon_{S}-d_{0}}{b_{S}}, 0\right)$ is unstable.

We next consider the asymptotic behavior of the combined dynamic system.

\section{Combined system under impulsive tagging}

We now combine Eqs. (29)-(30) and (33)-(34), incorporating the impulsive tagging at $x=0, t_{k}=k \Delta t, k=1,2,3, \ldots$. Recall that $N=S-T$ and $z=x-c\left(t-t_{0}\right)$. We thus arrive at the following impulsive system at $x=0$ :

$$
\begin{aligned}
& \frac{d X}{d z}=Y, \\
& \frac{d Y}{d z}=\frac{d_{0}-b_{T}}{\rho} X-\frac{(c-u)}{\rho} Y, \\
& \frac{d U}{d z}=V \\
& x=0, t \neq t_{k}, k=1,2,3, \ldots, \\
& \frac{d V}{d z}=\frac{d_{0}-b_{S} \varepsilon_{S}}{\rho} U+\frac{c b_{S}}{\rho} U^{2}-\frac{(c-u)}{\rho} V \\
& \Delta X\left(z^{+}\right)=c p U(z)-c p X(z), \\
& \Delta Y\left(z^{+}\right)=0 \text {, } \\
& \Delta U\left(z^{+}\right)=0, \\
& x=0, t=t_{k}, k=1,2,3, \ldots . \\
& \Delta V\left(z^{+}\right)=0
\end{aligned}
$$


Since the level of untagged fish $n$ follows directly once we know the levels of tagged and total populations with $n(z)=s(z)-\tau(z)$, it suffices for us to make sure that

$$
n(z)=s(z)-\tau(z) \geq 0 .
$$

From the derivative of $s(z)$ we immediately see that $s(z)$ is strictly decreasing for all $z \geq 0$. On the other hand, $\tau(z)$ may initially increase until it reaches its maximum value at the point where $z=z_{M}$ at which

$$
\tau^{\prime}=\frac{d}{d z}\left[\left(C z+\tau_{0}\right) e^{-D z}\right]=-D\left(C z+\tau_{0}\right) e^{-D z}+C e^{-D z}=0
$$

giving

$$
z=z_{M}=\frac{C-D \tau_{0}}{C D}
$$

which is positive if

$$
\tau_{0}<C / D
$$

and the maximum value of $\tau(z)$ is

$$
\tau\left(z_{M}\right)=\frac{C}{D} e^{-1+D \tau_{0} / C}
$$

Thus, we will be ensured that $n(z)=s(z)-\tau(z)>0$ for all $z$ if we require $\tau\left(z_{M}\right)<s\left(z_{M}\right)$ and $\tau^{\prime}\left(z_{M}\right)<s^{\prime}\left(z_{M}\right)$. These can be both accomplished by $C$ being sufficiently small, which means the density of tagged fish should increase sufficiently slowly initially.

Considering (3), we see that the density of untagged fish is reduced by a fraction $p$ of its levels at $k \Delta t, k=1,2,3, \ldots$, while the density of tagged fish is incremented at the same amount at the same time. Thus, we can directly write the following solution for all $t$ :

$$
\begin{aligned}
S(x, t) & =\frac{A^{2} e^{-A\left(x-c\left(t-t_{0}\right)\right)}}{\left[\kappa+B e^{-A\left(x-c\left(t-t_{0}\right)\right) / 2}\right]^{2}}, \\
T(x, t) & =\left[C\left(x-c\left(t-t_{0}\right)\right)+\tau_{0}^{k}\right] e^{-D\left(x-c\left(t-t_{0}\right)\right)}, \quad(k-1) \Delta t<t \leq k \Delta t,
\end{aligned}
$$

and

$$
N(x, t)=S(x, t)-T(x, t)
$$

where

$$
\begin{aligned}
\tau_{0}^{1} & =\tau_{0}=\tau, \\
\tau_{0}^{k} & =\tau_{0}^{k-1}+p N(0,(k-1) \Delta t) e^{-D c\left((k-1) \Delta t-t_{0}\right)}, \quad k=2,3,4, \ldots
\end{aligned}
$$

for which the parameters are related according to (11), (12), (19), (20), and (23), subject to the conditions (21) and (22). 
Based on the above solution, to ensure that the stock of tagged fish is not depleted at the tagging station $x=0$, so that $T(x, t)>0$, we need

$$
\left.-c C\left(k \Delta t-t_{0}\right)\right)+\tau_{0}^{k}>0
$$

where $\tau_{0}^{k}$ is given by (43). This is still conditional on our prior knowledge of the untagged fish density. We then use the fact that $N(x, t)=S(x, t)-T(x, t)$, where $s(z)$ is an increasing function and $\tau(z)$ is decreasing as $t \rightarrow \infty$ (z becoming more and more negative) at a fixed $x$, to find that

$$
N(x, k \Delta t) \geq s_{0}-\tau_{0}, \quad k=1,2,3, \ldots
$$

Thus, iteratively,

$$
\begin{aligned}
\tau_{0}^{k} & =\tau_{0}^{k-1}+p N(0,(k-1) \Delta t) e^{-D\left(c\left((k-1) \Delta t+t_{0}\right)\right)}, \\
\tau_{0}^{k} & =\tau_{0}^{k-2}+p N(0,(k-2) \Delta t) e^{-D c\left((k-2) \Delta t-t_{0}\right)}+p N(0,(k-1) \Delta t) e^{-D c\left((k-1) \Delta t-t_{0}\right)}, \\
\vdots & \\
\tau_{0}^{k} & =\tau_{0}^{1}+p \sum_{i=1}^{k-1} N(0,(k-i) \Delta t) e^{-D c\left((k-i) \Delta t-t_{0}\right)}, \\
\tau_{0}^{k} & \geq \tau_{0}+p \sum_{i=1}^{k-1}\left(s_{0}-\tau_{0}\right) e^{-D c(k-i) \Delta t}=\tau_{0}+\frac{p\left(s_{0}-\tau_{0}\right) e^{-D c \Delta t}\left(1-e^{-D c(k-i) \Delta t}\right)}{1-e^{-D c \Delta t}} \\
& \geq \tau_{0}+p\left(s_{0}-\tau_{0}\right) e^{-D c \Delta t} .
\end{aligned}
$$

This leads us to the following lower bound $\underline{p}$ of the fraction of the untagged population that we should tag so that the stock of tagged fish is sustainable:

$$
p>\left(\frac{\left.c C\left(k \Delta t-t_{0}\right)\right)-\tau_{0}}{s_{0}-\tau_{0}}\right) e^{D c \Delta t} \equiv \underline{p}
$$

while the upper bound $\overline{\Delta t}$ of the length of the time period we might go without tagging is, by (44),

$$
\Delta t<\frac{1}{k}\left(t_{0}+\frac{\tau_{0}^{k}}{c C}\right) \equiv \overline{\Delta t}
$$

\section{Discussion and conclusion}

Figure 1 shows the plots of total fish density $s$, tagged fish density $\tau$ and untagged fish density $n$ as functions of spatial dimension $x$ for different time $t$ between 0 and 6 , as traveling waves moving from left to right. Here, $c=0.1, A=1, B=1, C=0.6, D=1.25, u=0.05$, $\tau_{0}=0.3, s_{0}=0.64, \kappa=0.25, p=0.2, \rho=0.02$, and $t_{0}=2.0$. These are analytical solutions of the system without impulsive tagging. We observe that the density of tagged fish rises initially, then decreases the further we move from the reference location $x=0$, while the total number $s$ decreases steadily with $x$. 


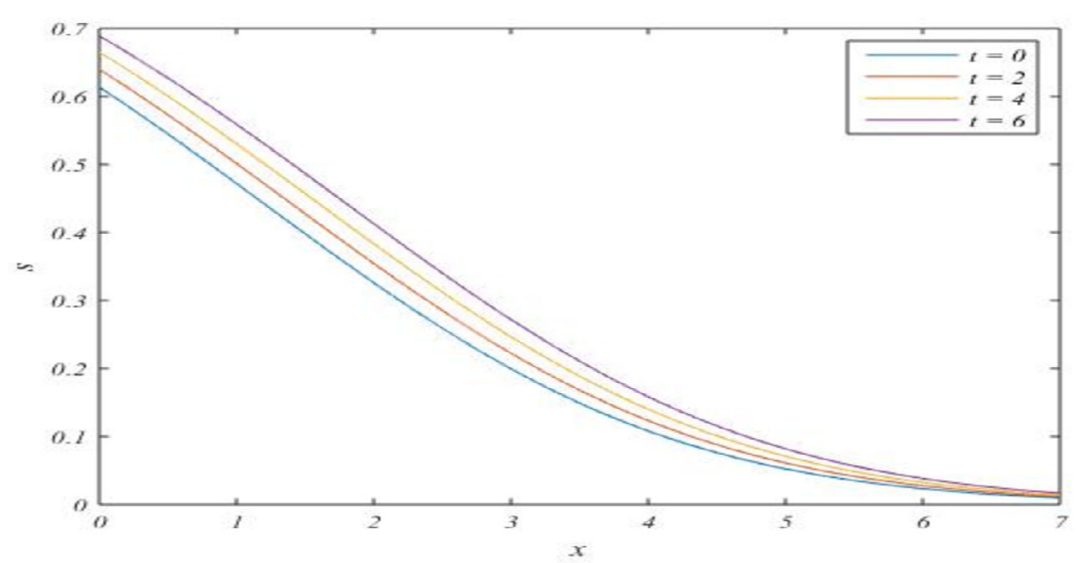

(a)

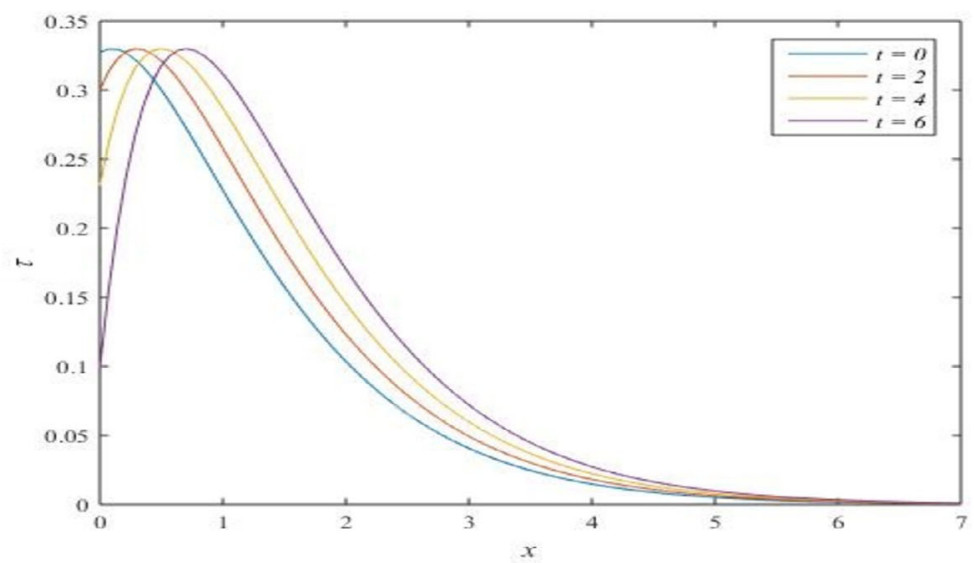

(b)

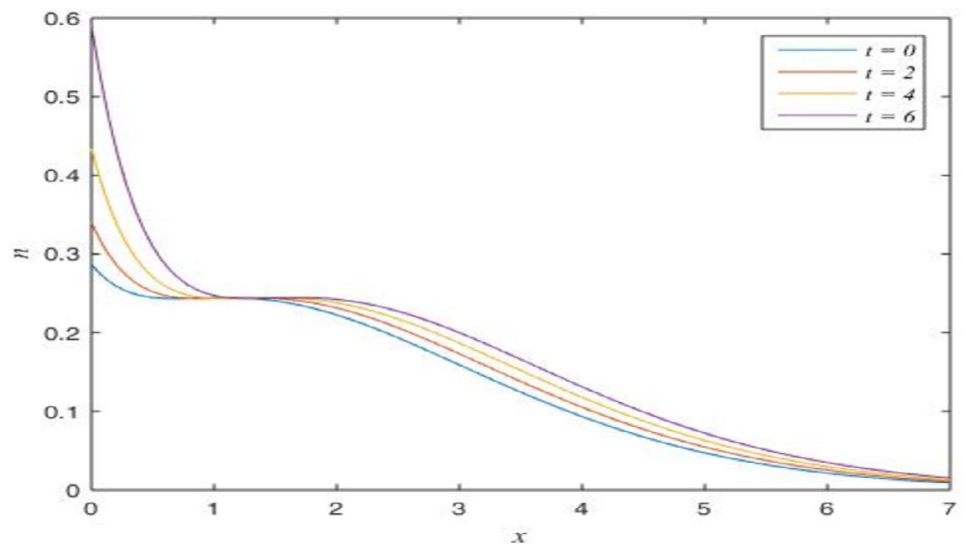

(c)

Figure 1 Traveling wave solution under no tagging. Plots of (a) total fish density $s$, (b) tagged fish density $\tau$, and (c) untagged fish density $n$ as functions of spatial dimension $x$ for different time $t$ between 0 and 6 , as traveling waves moving from left to right. Here, $c=0.1, A=1, B=1, C=0.6, D=1.25, u=0.05, \tau_{0}=0.3$, $s_{0}=0.64, \kappa=0.25, p=0.2, \rho=0.02$, and $t_{0}=2.0$

We also observe that, as time passes, the wave (or school) of tagged fish moves away so that there will be less and less tagged fish at the starting position, until there would be none left unless they are replenished. Impulsive tagging creates new stocks of tagged fish 


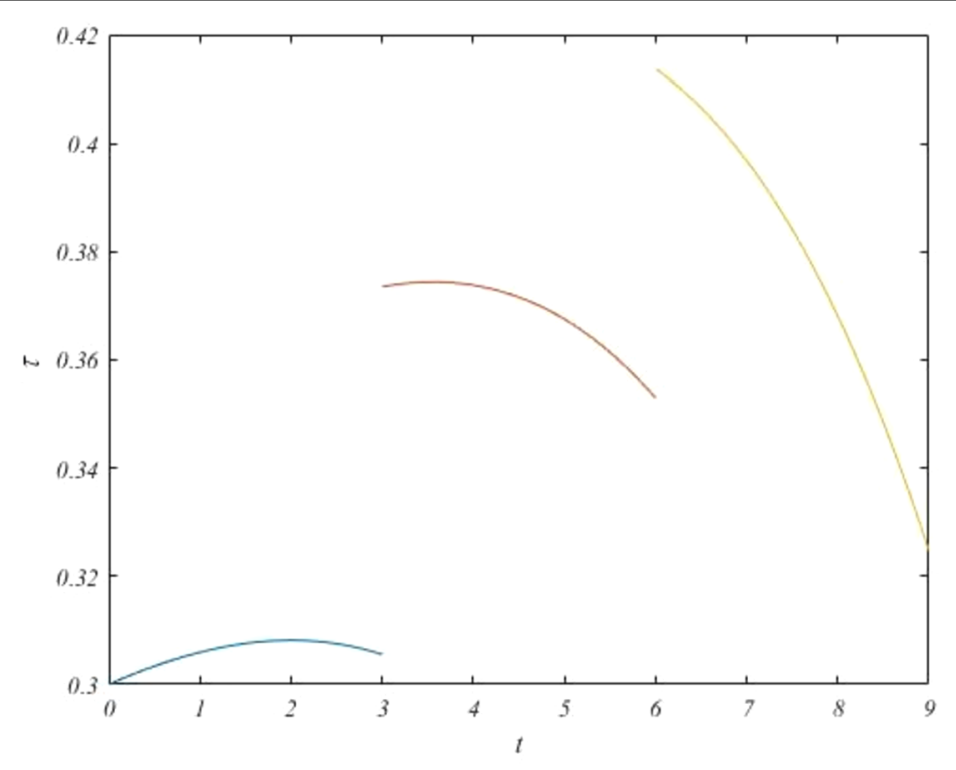

Figure 2 Solution with impulsive tagging. Time series of the density of tagged fish at the spatial position $x=0$, with impulsive tagging at the times $t=k \Delta t, \Delta t=3, k=1,2,3,4, \ldots$. Here, $c=0.1, A=1, B=1, C=0.3$, $D=1.25, u=0.05, \tau_{0}=0.3, s_{0}=0.64, \kappa=0.25, p=0.2, \rho=0.02$, and $t_{0}=0$

to replace the leaving swarms of fish which have been tagged earlier. This is modeled by the impulsive system (1)-(3). We show in Fig. 2 the time series of the density of tagged fish at the spatial position where $x=0$, corresponding to the analytical solution of the model system (1)-(3) with impulsive tagging, where the jumps in the level of tagged fish density are observed as expected at the times $t=k \Delta t, \Delta t=3, k=1,2,3,4, \ldots$ Here, $c=0.1, A=1$, $B=1, C=0.3, D=1.25, u=0.05, \tau_{0}=0.3, s_{0}=0.64, \kappa=0.25, p=0.2, \rho=0.02$, and $t_{0}=0$.

We show a 3-dimensional plot of this impulsive case in Fig. 3 so that we are able to see how the tagged fish density develops as we move in the spatial direction as well as when time progresses. With these parametric values, the number of tagged fish will grow as time passes as we keep replenishing it every $\Delta t=3$ units in time. Typically, $t$ could be measured in weeks, which means tagging is done every 3 weeks or so in this time series plot. If tagging is spaced out more in time, we can expect the tagged fish stock to vanish quickly. On the other hand, we have given conditions under which impulsive tagging ensures that fish stocks do not get depleted as time passes.

We observe, from the traveling wave solution that we have derived and plotted in these figures, that both tagged and non-tagged fish populations diminish to zero far downstream, which justifies our assumption made on using the linear term for the continuous tagging effect in our model.

It is extremely difficult to keep track of and manage fisheries especially with the highly mobile aquatic species. Utilizing Marine Protected Areas (MPAs) has been effective for many relatively sluggish marine species, but it poses many limitations in testing and verification when dealing with highly mobile species due to their frequent movement outside the protected area. In order to protect marine lives, restore biomass, and increase fishery yields, a model such as ours can help to overcome these limitations by identifying designs and predict potential outcomes. 


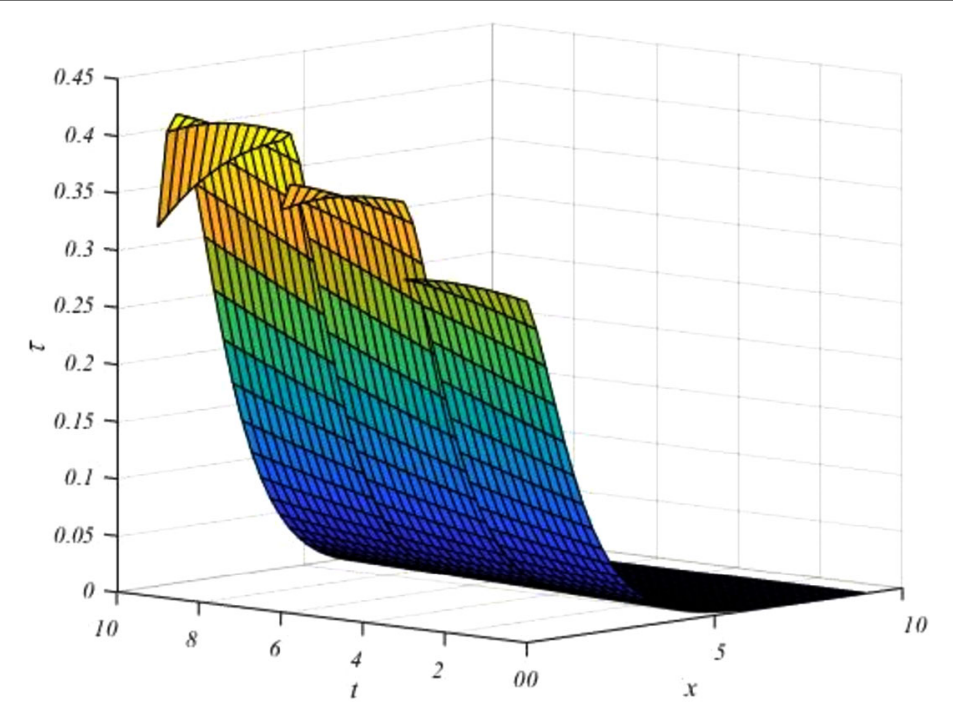

Figure 3 Three-dimensional view. 3D plot of tagged fish density with impulsive tagging. Here, $t=k \Delta t$, $\Delta t=3, k=1,2,3,4, \ldots, C=0.1, A=1, B=1, C=0.3, D=1.25, u=0.05, \tau_{0}=0.3, s_{0}=0.64, \kappa=0.25, p=0.2$, $\rho=0.02$ and $t_{0}=0$

Tagging and marking of fish populations have become a common method used to examine movement patterns and estimate growth rates, as well as other parameters of interest including their abundances. For the tagging study to be effective, careful planning is needed to ensure that the tagging study's objectives are met. Among the most important considerations is the necessity to ensure that sufficient numbers of animals are marked and recaptured to generate parameter estimates of sufficient precision to meet management needs. Increases in the precision of estimates deriving from the analytical study of both tagged population, continuous and/or impulsive, and untagged population may be possible by stochastic simulations without much difficulty. Employing appropriate analytical methods, with the help of appropriate models providing valuable insight, would ensure that the results are interpreted properly and accurately. Our model is, therefore, expected to shed lights on how fish movement dynamics vary with the physical parameters, which affects the design of effective alternatives to managing highly mobile stocks in the open waters.

Funding

We acknowledge the support of the Centre of Excellence in Mathematics, the Commission on Higher Education, Thailand.

Competing interests

The authors declare that they have no competing interests.

Authors' contributions

All authors contributed equally to this work. All authors read and approved the final manuscript.

Author details

'Department of Mathematics, Faculty of Science, Mahidol University, Bangkok, Thailand. ${ }^{2}$ Department of Mathematics, Faculty of Applied Science, King Mongkut's University of Technology North Bangkok, Bangkok, Thailand. ${ }^{3}$ Centre of Excellence in Mathematics, CHE, Bangkok, Thailand.

\section{Publisher's Note}

Springer Nature remains neutral with regard to jurisdictional claims in published maps and institutional affiliations. 


\section{References}

1. Hayden, T.A., Holbrook, C.M., Fielder, D.G., Vandergoot, C.S., Bergstedt, R.A., Dettmers, J.M., Krueger, C.C., Cooke, S.J.: Acoustic telemetry reveals large-scale migration patterns of Walleye in Lake Huron. PLoS ONE (2014) https://doi.org/10.1371/journal.pone.0114833

2. Sibert, J.R., Hampton, J., Fournier, D.A., Bills, P.J.: An advection-diffusion-reaction model for the estimation of fish movement parameters from tagging data, with application to skipjack tuna (Katsuwonus pelamis). Can. J. Fish. Aquat. Sci. 56, 925-938 (1999)

3. Stephenson, R.L.: Stock complexity in fisheries management: a perspective of emerging issues related to population sub-units. Fish. Res. 43, 247-249 (1999)

4. Ying, Y., Chen, Y., Lin, L., Gao, T.: Risks of ignoring fish population spatial stock structure. Can. J. Fish. Aquat. Sci. 68, 2101-2120 (2011)

5. Guan, W., Cao, J., Chen, Y., Cieri, M.: Impacts of population and fishery spatial structures on fishery stock assessment. Can. J. Fish. Aquat. Sci. 70, 1178-1189(2013)

6. Hutchings, J.A.: Collapse and recovery of marine fisheries. Science $406,882-885$ (2000)

7. Cadrin, S.X., Secor, D.H.: Accounting for spatial population structure in stock assessment: past, present, and future. In: The Future of. Fisheries Science in North America. Fish \& Fisheries Series, pp. 405-426. Springer, New York (2009)

8. Hilborn, R.: Determination of fish movement patterns from tag-recoveries using maximum likelihood estimators. Can. J. Fish. Aquat. Sci. 47, 635-643 (1990)

9. Vandergoot, C.S., Brenden, T.O.: Accuracy and precision of fishery and demographic estimates from a spatial tag-recovery model when inter-regional movements are treated as fixed. Fish. Res. 164, 8-25 (2015)

10. Pine, W.E., Pollock, K.H., Hightower, J.E., Kwak, T.J., Rice, J.A.: A review of tagging methods for estimating fish population size and components of mortality. Fisheries 28, 10-23 (2003)

11. Adam, M.S., Sibert, J.R.: Population dynamics and movements of skipjack tuna (Katsuwonus pelamis) in the Maldivian fishery: analysis of tagging data from an advection-diffusion-reaction model. Aquatic Living Resour. 15, 13-23 (2002)

12. Bertignac, M., Lehodey, P., Hampton, J.: A spatial population dynamics simulation model of tropical tunas using habitat index based on environmental parameters. Fish. Oceanogr. https://doi.org/10.10462/j.1365-2419.00065.x

13. Faugeras, B., Maury, O.: An advection-diffusion-reaction size-structured fish population dynamics model combined with a statistical parameter estimation procedure: application to the Indian Ocean skipjack tuna fishery. Math. Biosci. Eng. 2, 1-23 (2005)

14. Boonrangsimana, S., Bunwonga, K., Moore, E.J.: A bifurcation path to chaos in a time-delay fisheries predator-prey model with prey consumption by immature and mature predators. Math. Comput. Simul. 124, 16-29 (2016)

15. Milewski, P.A., Yang, X.: A simple model for biological aggregation with symmetric sensing. Commun. Math. Sci. 6 397-416 (2008)

16. Birnir, B., Maury, O.: An ODE model of the motion of pelagic fish. J. Stat. Phys. 128, 1-34 (2007)

\section{Submit your manuscript to a SpringerOpen ${ }^{\circ}$ journal and benefit from:}

- Convenient online submission

- Rigorous peer review

- Open access: articles freely available online

- High visibility within the field

- Retaining the copyright to your article

Submit your next manuscript at $\boldsymbol{~ s p r i n g e r o p e n . c o m ~}$ 